\title{
Cobalt (III)-EDTA dissociates and chromium (III)-EDTA is slightly more stable under in vitro reducing conditions comparable to those in the rumen
}

\author{
Peter J. Van Soest ${ }^{1 *}$ and Mary Beth Hall ${ }^{2} \dagger$ (1) \\ ${ }^{1}$ Department of Animal Science, Cornell University, Ithaca, NY 14853 \\ ${ }^{2}$ U.S. Dairy Forage Research Center, USDA-Agricultural Research Service, Madison, WI 53706
}

\begin{abstract}
Ideal digesta markers used for feeding studies are inert, unabsorbable, and move with the digesta they are intended to mark. Both chromium (III) and cobalt (III) salts of EDTA (CrEDTA and CoEDTA, respectively) are used as markers of liquid digesta in dairy cattle research. A small portion is absorbed and excreted in urine, but the markers are assumed to remain unreactive and as inert salts in the digesta and animal. The degree to which these colored salts remain bound in solution can be estimated through spectrophotometric measurement at their wavelength $(\lambda)$ of peak absorbance. The objective of this in vitro study was to evaluate whether CrEDTA and CoEDTA dissociate under reducing conditions that could be experienced in the rumen. In a completely randomized design with 2 replicate analytical runs and samples in duplicate within run, approximately $26 \mathrm{mg} / \mathrm{L} \mathrm{Cr}$ from CrEDTA or Co from CoEDTA was incubated in a 26$\mathrm{mL}$ reaction volume containing $20 \mathrm{~mL}$ of Goering and Van Soest medium without tryptone, $3 \mathrm{~mL}$ of CoEDTA or CrEDTA solutions, or water (reagent blanks), and $3.0 \mathrm{~mL}$ of a combination of distilled water with $0,0.25$, $0.50,0.75$, or $1.00 \mathrm{~mL}$ of reducing solution (RedSol). After incubation for $0.5 \mathrm{~h}$ at $39^{\circ} \mathrm{C}$, absorbance was read at $\lambda=535,465$, and $560 \mathrm{~nm}$, the peak $\lambda$ for EDTA salts of $\mathrm{Co}(\mathrm{III}), \mathrm{Co}(\mathrm{II})$, and $\mathrm{Cr}(\mathrm{III})$, respectively. Mean reagent blank values were subtracted from CoEDTA and CrEDTA data. The absorbance data at peak $\lambda$ were analyzed by marker in models that included RedSol with analytical run as a random variable. Contrasts were used to detect linear through quartic effects of RedSol. Samples with RedSol had redox potentials of -250 to $-328 \mathrm{mV}$, which are within the range of reported ruminal measures. As RedSol increased, CoEDTA showed a linear decline of $75 \%$ in ABS at 535
\end{abstract}

Received May 22, 2020.

Accepted July 13, 2020.

*Professor Emeritus.

†Corresponding author: marybeth.hall@ars.usda.gov $\mathrm{nm}$ and a quadratic 4-fold increase followed by a $60 \%$ decline at $465 \mathrm{~nm}$. These responses indicate a reduction of $\mathrm{Co}(\mathrm{III})$ to $\mathrm{Co}(\mathrm{II})$ and subsequent dissociation of $\mathrm{Co}$ (II)EDTA. The absorbance of CrEDTA at $560 \mathrm{~nm}$ showed a tendency for an $8 \%$ linear decrease as RedSol increased. Wavescans from $\lambda=330$ to $700 \mathrm{~nm}$ showed CrEDTA retaining its characteristic 2-peak pattern as RedSol increased, whereas CoEDTA curves deformed entirely. We conclude that CoEDTA is not a stable, inert digesta marker under reducing conditions achievable in the rumen and is therefore unsuitable for use in studies with ruminants. Reexamination of the suitability of available liquid digesta markers is advised.

Key words: CoEDTA, CrEDTA, reducing conditions, liquid digesta, marker

\section{INTRODUCTION}

Studies with lactating dairy cattle have used markers for liquid digesta to estimate liquid passage, rumen volume, and ruminal substrate disappearance (Weisbjerg et al., 1998; Shingfield et al., 2008). Ideal digesta markers for use in feeding studies are indigestible, unabsorbable, move with the portion of the digesta they are intended to mark (Faichney, 1993), and should be accurately and specifically measurable. However, all markers have limitations. The EDTA salts of chromium (III) (CrEDTA) and cobalt (III) (CoEDTA) were developed as liquid markers to allow greater specificity of analysis, with CoEDTA introduced as a non-Cr-containing material to facilitate use of multiple markers (Udén et al., 1980). They are among the most commonly used liquid markers. Small portions of both markers are excreted in urine (Shingfield et al., 2008), which violates the criterion of digesta markers being unabsorbable. A greater concern that has not been well explored is the potential for the metal-EDTA markers to go from their inert salt forms to dissociated ions that may be reactive within the gut or when absorbed by the animal. Shingfield et al. (2008) demonstrated that ruminal infusion of CoEDTA but not CrEDTA resulted in changes in the profile of fatty acids in milk, 
possibly via effects on desaturase enzymes in the cow's tissues. Such findings raise serious concerns regarding the question of marker dissociation and its effects in the conduct of dairy cattle research. Approximately $4 \%$ of CoEDTA and up to $14 \%$ of CrEDTA has been shown to dissociate in autoclaved, clarified rumen fluid that was not maintained in a reduced state, with differences detected between rumen fluid sources from 2 different cows (Hall and Van Soest, 2019b). Transfer of the metal ions to other soluble components in the rumen fluid appears to be the likely basis for the decline in detectable markers.

The effect of reducing conditions in the rumen environment on metal-EDTA stability has not been explored. The CoEDTA and CrEDTA prepared as markers incorporate the trivalent metal ions $\mathrm{Co}$ (III) and $\mathrm{Cr}(\mathrm{III})$. The use of hydrogen peroxide in the synthesis of CoEDTA (Udén et al., 1980) converts Co(II) to $\mathrm{Co}(\mathrm{III})$, which greatly increases the stability of the CoEDTA marker; the stability constants of $\mathrm{Co}(\mathrm{II})$ EDTA, Co(III)EDTA, and Cr(III)EDTA are 16.5, 41.5, and 23.4, respectively (Anderegg, 1977). Reduction of EDTA-associated $\mathrm{Co}(\mathrm{III})$ to $\mathrm{Co}(\mathrm{II})$ is reported to occur at oxidation-reduction potentials ( ORP) of -60 to $-68 \mathrm{mV}$ (Ogino and Ogino, 1983), which are less negative than ORP achieved in the rumen in vivo $(-231$ to $-480 \mathrm{mV}$; Waghorn, 1991; Richter et al., 2010 from figure data; Qin et al., 2017). Variation in ruminal ORP reported in the literature may be related to sample handling, with ORP reported to be less negative for rumen fluid samples processed outside the rumen than for those measured continuously in vivo with indwelling wireless probes in the rumen (Huang et al., 2018). It appears that diet can affect ruminal ORP. If $\mathrm{Co}$ (III) EDTA were reduced to $\mathrm{Co}(\mathrm{II}) \mathrm{EDTA}$ under ruminal conditions, the marker would be more prone to dissociation.

A challenge to addressing the question of marker dissociation is the issue of measuring dissociation of a salt in ruminal digesta. In such a complex matrix, it is typically not feasible to measure a compound as an ionically bound salt. However, because CrEDTA and CoEDTA in their bound forms are colored compounds with distinct absorbance patterns across wavelengths, and have absorbance maxima at specific wavelengths, they can be detected spectrophotometrically (Hamm, 1953; Paraneeiswaran et al., 2015). Spectrophotometry has been used to monitor progression of CrEDTA formation in kinetic studies (Hedrick, 1965). Accordingly, measurement of the absorbance of light at specific wavelengths and across wavelengths of visible light can be used to evaluate whether Cr- and CoEDTA are present in their ionically bound, colored forms, or whether they have dissociated. Additionally, the wavelength of maximum absorption of Co(III)EDTA (535 nm) differs from that of Co(II)EDTA (465 nm) (Xue and Traina, 1996), allowing monitoring of the conversion of the species. The wavelengths of maximum absorption can be slightly affected by $\mathrm{pH}$, with maximum absorption for CrEDTA occurring at $541 \mathrm{~nm}$ at slightly acidic $\mathrm{pH}$ (Cerar, 2015).

The objective of this study was to determine whether CrEDTA and CoEDTA salts dissociate under reducing conditions in vitro that are comparable to reducing conditions experienced in the rumen. The use of reduced medium rather than rumen fluid with microbes removed the effect of microbial action; the effect of other components present in rumen fluid have been evaluated previously (Hall and Van Soest, 2019b). This approach allowed us to focus the experiment on the effects of reducing conditions and provided solutions that were free of particulate matter and microbes that would interfere with absorbance measurements.

\section{MATERIALS AND METHODS}

The lithium salt of Cr(III)EDTA was prepared using Cr(III) acetate hydroxide (Hall and Van Soest, 2019a), and that of Co(III)EDTA prepared according to the method of Udén et al. (1980). Solutions of Cr(III) EDTA $(1.0286 \mathrm{~g} / 500 \mathrm{~mL})$ and Co(III)EDTA (0.9260 $\mathrm{g} / 500 \mathrm{~mL}$ ) were prepared with distilled water. Marker concentrations were set so that when mixed with medium, they provided a concentration equivalent to approximately $26 \mathrm{mg}$ of $\mathrm{Cr}$ or $\mathrm{Co} / \mathrm{L}$.

\section{Sample Preparation}

Two incubation runs were performed under anaerobic conditions, with Co(III)EDTA and Cr(III)EDTA included in each run. Goering and Van Soest (1970) media were prepared with no tryptone added and with or without resazurin. The media were prepared the day before an incubation run, sparged for $1 \mathrm{~h}$ with $\mathrm{CO}_{2}$, the flasks stoppered, and stored at $4^{\circ} \mathrm{C}$ until used the following morning. Incubations were performed in sealed borosilicate glass fermentation tubes $(121 \mathrm{~mm}$ long, 28 $\mathrm{mm}$ outer diameter, $2.8 \mathrm{~mm}$ wall thickness, with the end of the tubes formed to be sealed with a crown cap; custom made by Wilmad-LabGlass, Vineland, NJ). Each marker $\times$ reducing solution $($ RedSol) treatment was prepared in duplicate in each run. Replication was based on repeatability of EDTA marker absorbance measures found in a previous investigation (Hall and Van Soest, 2019b). Each tube contained $20 \mathrm{~mL}$ of medium, $3 \mathrm{~mL}$ of marker solution, and $3 \mathrm{~mL}$ of a combination of distilled water and RedSol, with the latter added at $0,0.25,0.50,0.75$, and $1.00 \mathrm{~mL}$. In each run, 
marker solutions and Goering and Van Soest (1970) medium with all solutions except RedSol were brought to $39^{\circ} \mathrm{C}$ in a water bath. After RedSol was added to all tubes, the headspace was gassed with $\mathrm{CO}_{2}$ and the tubes were capped with crown caps, inverted to mix, and incubated for $0.5 \mathrm{~h}$ at $39^{\circ} \mathrm{C}$ in a water bath.

After incubation, the color of each tube with resazurin was recorded as an indicator of reduction. Each tube without resazurin was inverted to mix, opened, the $\mathrm{pH}$ was measured, and $200 \mu \mathrm{L}$ of solution was pipetted in duplicate into wells of a 96-well plate using a positive displacement repeat pipetter (Eppendorf Repeater M4, Eppendorf North America, Hauppauge, NY), giving an approximately $5-\mathrm{mm}$ path length. A plate reader was used to allow all samples to be read in less time than would be possible with a spectrophotometer with cuvettes. Once all solutions were pipetted, the absorbances were read in a plate reader spectrophotometer (EONC, Biotek Instruments Inc., Winooski, VT) at wavelengths of 330 to $700 \mathrm{~nm}$ in 10-nm increments, and at 465,535 , and $541 \mathrm{~nm}$. Values for replicate wells per sample in the plate reader were averaged for statistical analysis. For each marker and RedSol treatment, the average of the appropriate RedSol reagent blanks containing no marker was subtracted from the values for the samples with marker added. The wavelength of numeric maximum absorbance for Co(III)EDTA and Cr(III)EDTA incubated with no RedSol was selected. Remaining solutions were transferred to scintillation vials and frozen at $-20^{\circ} \mathrm{C}$ for future analysis. The sample solutions from each run were analyzed for minerals by inductively coupled plasma spectroscopy (Official Method 990.08, AOAC International, 2005; model 5100, Agilent Technologies, Santa Clara, CA).

Evaluation of the effect of RedSol dose on ORP was conducted in a separate, single analytical run using duplicate samples prepared with and without resazurin as in the experiment but with no markers added. Samples were measured with an ORP electrode (Mettler Toledo InLab Redox combo electrode, P/N-51343200, VWR, Batavia, IL) on a $\mathrm{pH}$ meter (Seven Easy $\mathrm{pH}$ meter, Mettler-Toledo, Scherzenbach, Switzerland). Samples were analyzed within 40 to 66 min of addition of RedSol. Upon decapping of tubes containing sample solution, the redox electrode was inserted to within $1 \mathrm{~cm}$ of the bottom of the tube and used to stir the samples. This left only a narrow band of liquid at the surface between the probe and tube to interact with air. The ORP was recorded when it reached an apparent minimum value. Redox buffer solution (220 mV, pH 7; \#51350060, Mettler-Toledo, Greifensee, Switzerland) used as a standard read as $228 \mathrm{mV}$ with the ORP probe. This offset value of $8 \mathrm{mV}$ was subtracted from the values determined on the samples.

\section{Statistical Analysis}

The study was performed as a completely randomized design with the individual tube on which a measurement was taken as the experimental unit. Analytical run was a random variable, and all other variables were fixed and treated as non-numeric classification variables. For the equally spaced RedSol doses $(0,0.25$, $0.50,0.75$, and $1.00 \mathrm{~mL}$ ), contrasts were used to evaluate linear through quartic patterns of response. With all observations made at a single incubation time, there were no repeated measures.

For the response variables of sample solution $\mathrm{pH}$ and mineral concentrations, the statistical models included analytical run, marker type [Co(III)EDTA, Cr(III) EDTA], RedSol dose, and the interaction of marker type and RedSol dose $(\mathrm{n}=40$, error $\mathrm{df}=29)$. Evaluation of ORP data was performed using data from a single analytical run in a model that included RedSol dose, presence or absence of resazurin, and the interaction term $(\mathrm{n}=20$, error $\mathrm{df}=10$ for all RedSol doses; with $0 \mathrm{~mL}$ RedSol omitted, $\mathrm{n}=16$, error $\mathrm{df}=8$ ). Absorbance responses to RedSol dose of Co(III)EDTA solutions at 535 and $465 \mathrm{~nm}$ and $\mathrm{Cr}$ (III)EDTA solutions at $560 \mathrm{~nm}$ were evaluated separately in models that included analytical run and RedSol dose; in each statistical analysis, $\mathrm{n}=20$, with 14 df for error. No statistics were applied to the wavescan data. Analyses were performed using the MIXED procedure of SAS (version 9.4, SAS Institute Inc., Cary, NC). Least squares means values are presented with standard errors of the difference (SED) unless otherwise stated. Significance was declared at $P<0.05$. Data were evaluated for normality using the Shapiro-Wilk evaluation in the NORMAL option in PROC UNIVARIATE of SAS, with the analysis performed on the residuals generated from the statistical models used. If needed, data were transformed to achieve normality with natural log, exponential, inverse, sine, cosine, and tangent transformations evaluated.

\section{RESULTS AND DISCUSSION}

\section{Normality of Data}

The data that were the focus of the experiment-absorbances for each marker at specific wavelengths as affected by reducing solution addition-were normally distributed, as shown by $P>0.05$ for the Shapiro-Wilk evaluation $[P=0.40$ for $\mathrm{Cr}(\mathrm{III})$ EDTA at $560 \mathrm{~nm} ; P>$ 0.93 for Co(III)EDTA at 535 and $465 \mathrm{~nm}$ ]. For data included as descriptors of run conditions, $\mathrm{pH}(P=$ $0.93)$, ORP $(P=0.85)$, and most minerals $(P=0.10$ to $0.70)$ were normally distributed. The 2 exceptions were 
Van Soest and Hall: LIQUID MARKER DISSOCIATION IN REDUCING CONDITIONS

Table 1. Mineral concentrations $(\mathrm{mg} / \mathrm{L})$ of marker (cobalt-EDTA or chromium-EDTA) by reducing solution treatments ${ }^{1}$

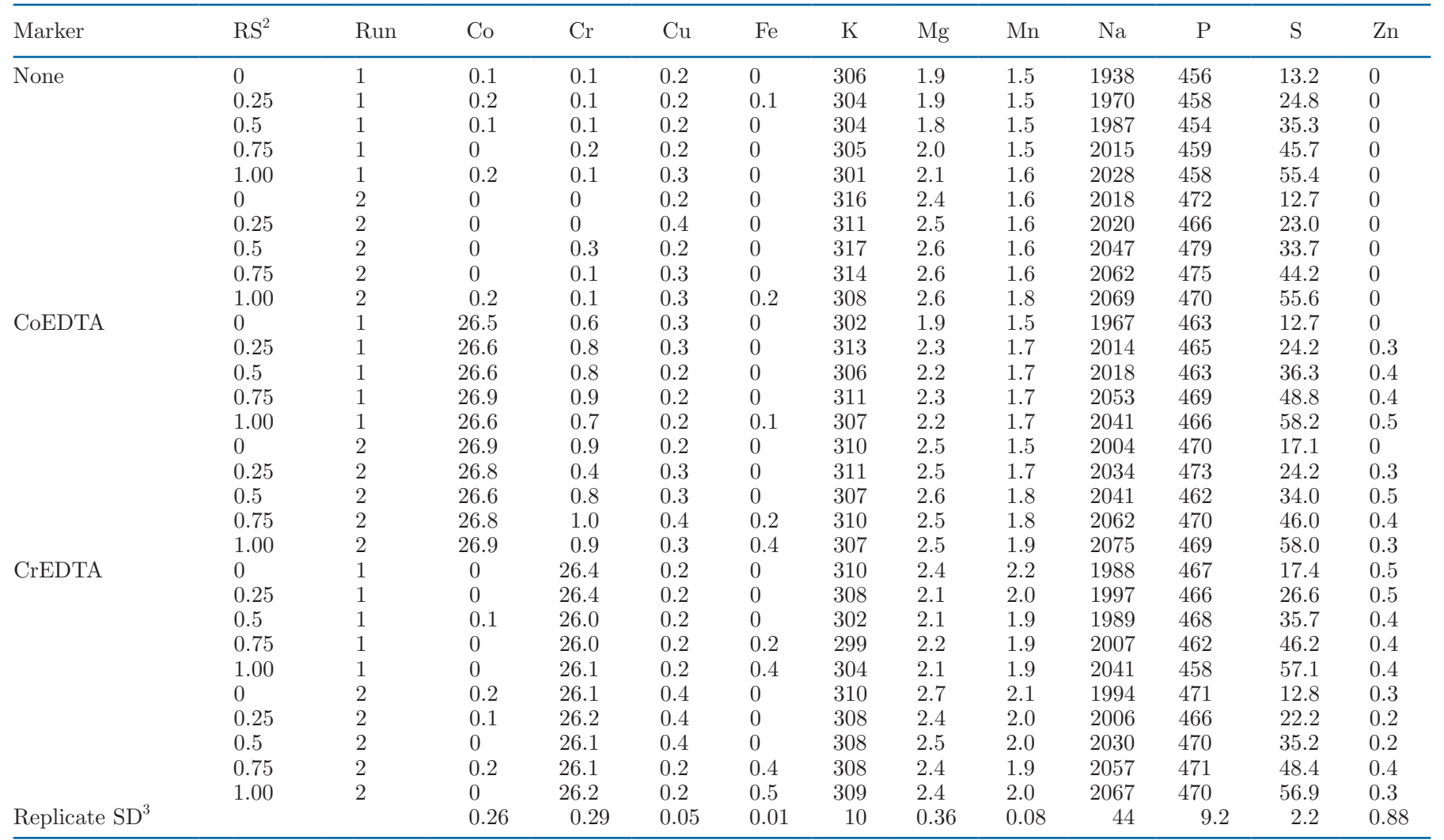

${ }^{1}$ Values are arithmetic means of duplicate treatment tubes within run.

${ }^{2} \mathrm{RS}=$ milliliters of reducing solution added.

${ }^{3}$ Average of the SD of replicate sample tubes within analytical run for a given mineral. This value gives an estimate of the precision of the analyses but is also affected by variation between sample tubes.

sample solution iron and manganese concentrations $(P$ $<0.01$ for both). Transformation of the manganese concentration data as its inverse $(1 / Y)$ normalized the data $(P>0.05)$ but did not change the $P$-value patterns of significance of treatments. It was not possible to normalize the iron data, likely because of the substantial number of $0 \mathrm{mg} / \mathrm{L}$ concentrations in that data, with values describing low concentrations from 0 to 0.6 $\mathrm{mg} / \mathrm{L}$. Accordingly, iron data are reported (Table 1) but not evaluated statistically.

\section{Run Conditions}

Measurements made on samples conserved at the end of the analytical runs indicated that comparable, targeted amounts of marker were added to each tube and that reagent blanks contained little $\mathrm{Co}$ or $\mathrm{Cr}$ (Table 1). Both sodium and sulfur concentrations increased linearly with increasing additions of RedSol $(P<0.01$ for both), reflecting contributions of the $\mathrm{NaOH}$, cysteine $\mathrm{HCl}$, and $\mathrm{Na}$ sulfide used in the RedSol formulation (Goering and Van Soest, 1970). There were no differ- ences in concentrations of copper $(P>0.50)$, potassium $(P>0.58)$, magnesium $(P>0.64)$, or phosphorus $(P>0.81)$ between the Co(III)EDTA and $\mathrm{Cr}(\mathrm{III})$ EDTA treatments, RedSol levels, or their interaction. Two of the minerals having the lowest concentrations, manganese and zinc, differed between markers $(P<$ 0.02 for both), and manganese differed by RedSol addition and its interaction with marker treatment $(P$ $<0.01$ for both). Although significant, the differences detected were $<1 \mathrm{mg}$ of mineral/L and likely caused by contamination in the original chemicals. Similarly, we attributed the $<1 \mathrm{mg}$ of $\mathrm{Cr} / \mathrm{L}$ detected in the $\mathrm{Co}$ (III) EDTA sample solutions to an unknown source of contamination. The $\mathrm{pH}$ of the sample solutions differed slightly between the 2 analytical runs $(6.95$ and $6.88, P$ $<0.01, \mathrm{SED}=0.012)$, but did not differ by marker $(P$ $=0.27)$, amount of RedSol added $(P=0.61)$, or their interaction $(P=0.64)$ (data not shown).

Solutions without markers became more reduced with increasing RedSol addition $(P<0.01)$ and with versus without resazurin $(P<0.01$; Table 2$)$. Solutions containing 0.25 to $1.0 \mathrm{~mL}$ of RedSol gave ORP values 
within the bounds of those measured ruminally in vivo for dairy cattle ( -231 to $-480 \mathrm{mV}$; Waghorn, 1991; Richter et al., 2010 from figure data; Qin et al., 2017), suggesting that the experimental conditions were within an appropriate range to evaluate the effect of reducing conditions that could be experienced in the rumen. The interaction of RedSol dose and resazurin usage became nonsignificant when the 0 -mL RedSol dose data were omitted. This was likely due to the very large ORP difference $(176 \mathrm{mV})$ between samples with and without resazurin at $0 \mathrm{~mL}$ of RedSol compared with the much smaller 10 to $20 \mathrm{mV}$ differences at other RedSol dosages. Resazurin used under ambient lighting has been shown to shorten, by hours, the time required to fully reduce culture medium (Fukushima et al., 2003) and is the likely reason for the more negative ORP values noted with resazurin in the present study. With the 0-mL RedSol data omitted, ORP responses were quadratic for increasing RedSol dosages $(P<0.01)$.

Resazurin-related color change was used qualitatively as a reduction indicator in sample solutions not used for absorbance readings in the main experiment. For reagent blanks with no marker added, those with $0 \mathrm{~mL}$ of RedSol stayed blue, the color of unreduced resazurin. Reducing solution dosed at $0.25 \mathrm{~mL}$ gave faint pink solutions, and solutions became colorless at greater RedSol volumes. Solutions with $\mathrm{Cr}$ (III)EDTA or Co(III) EDTA added showed the same color pattern with some exceptions. The purple color of $\mathrm{Cr}$ (III)EDTA was evident in all tubes in which it was included, even with the resazurin color changes. The reddish purple color of $\mathrm{Co}$ (III)EDTA treatments was evident in solutions with 0 or $0.25 \mathrm{~mL}$ of RedSol, but took on a brown rather than clear color with greater RedSol additions, whether or not resazurin was present. This would indicate that changes in Co(III)EDTA, not in the resazurin, were the source of the brown color formation.

\section{Marker Response to Reducing Conditions}

Colored compounds in solution have characteristic wavelengths at which they achieve maximal absorbance values in spectrophotometric evaluations. Changes in absorbance at these wavelengths are the basis for detecting increasing and decreasing concentrations of analytes in colorimetric assays, as well as for estimating evolution (Hamm, 1953) or, conversely, destruction of a compound of interest. It must be noted that, although there is a wavelength of maximal absorbance for a particular compound or specie, that material also exhibits light absorbance at many other wavelengths, albeit to a lesser degree. Colored compounds have characteristic patterns of absorption across wavelengths that are modified by changes in the valence of associated metal ions or other compositional modifications of the compound (Xue and Traina, 1996).

The Co- and Cr-EDTA markers tested responded very differently to increasingly reduced conditions. The Co(III)EDTA showed a $75 \%$ decline in absorbance at its maximum absorbance wavelength of $535 \mathrm{~nm}$ (RedSol effect, $P<0.0001$, SED $=0.008$; patterns of response: linear, $P<0.0001$; quadratic, $P=0.07$; cubic, $P=0.03$; quartic, $P=0.47$; Figure 1a). Absorbances measured at $465 \mathrm{~nm}$, the wavelength of maximal absorption for the reduced Co(II)EDTA, showed a 4 -fold increase between 0 and $0.5 \mathrm{~mL}$ of RedSol before declining by $61 \%$ (RedSol effect, $P<0.0001$, SED $=0.009$; patterns of response: linear, $P=0.0033$; quadratic, $P<0.0001$; cubic, $P=$ 0.16 ; quartic, $P=0.10)$. Under the reducing conditions imposed, it appears that a substantial portion, if not

Table 2. Redox potentials $(\mathrm{mV})$ of media without and with resazurin ${ }^{1}$

\begin{tabular}{|c|c|c|c|c|}
\hline \multirow{2}{*}{$\begin{array}{l}\text { Reducing solution, } \\
\text { mL }\end{array}$} & \multicolumn{2}{|c|}{ No resazurin } & \multicolumn{2}{|c|}{ With resazurin } \\
\hline & Mean & $\mathrm{SD}$ & Mean & $\mathrm{SD}$ \\
\hline 0 & 196 & 3.5 & 20.5 & 3.54 \\
\hline 0.25 & -250 & 2.83 & -272 & 4.24 \\
\hline 0.50 & -285 & 5.66 & -304 & 7.07 \\
\hline 0.75 & -314 & 4.24 & -326 & 0.71 \\
\hline \multirow[t]{2}{*}{1.00} & -328 & 0.71 & -341 & 0 \\
\hline & & \multicolumn{3}{|c|}{$P$-value } \\
\hline Analysis & $\mathrm{SED}^{2}$ & $\begin{array}{l}\text { Reducing } \\
\text { solution, mL }\end{array}$ & $\begin{array}{l}\text { Resazurin } \\
\text { inclusion }\end{array}$ & Interaction \\
\hline All data & 3.90 & $<0.01$ & $<0.01$ & $<0.01$ \\
\hline Omitting $0 \mathrm{~mL}$ data & 3.98 & $<0.01$ & $<0.01$ & 0.34 \\
\hline
\end{tabular}


all, of the Co(III) associated with EDTA was reduced to $\mathrm{Co}$ (II); the absolute proportion of $\mathrm{Co}$ (III)EDTA reduced could not be determined because Co(III)EDTA normally shows some absorbance at $465 \mathrm{~nm}$ (see $0-\mathrm{mL}$ RedSol curve for Co(III)EDTA in Figure 2a), and $\mathrm{Co}$ (II)EDTA has some absorbance at $535 \mathrm{~nm}$ (Xue and Traina, 1996). With the lesser stability of Co(II)EDTA compared with $\mathrm{Co}$ (III) EDTA (stability constants of 16.5 and 41.5, respectively; Anderegg, 1977), we interpreted the increase and then decline in absorbance at $465 \mathrm{~nm}$ as feasibly representing generation of and subsequent dissociation of Co(II)EDTA. This scenario was supported by the pattern of absorbance for the Co(III)EDTA treatment across wavelengths from 330 to $700 \mathrm{~nm}$ (Figure 2a). Whereas at $0 \mathrm{~mL}$ RedSol, the absorbances show the pattern across wavelengths characteristic of Co(III)EDTA (Xue and Traina, 1996), the patterns deform with increasing doses of RedSol, with absorbances at shorter wavelengths increasing and those at $535 \mathrm{~nm}$ declining. The gross alteration of the absorbance pattern is indicative of the conversion of Co(III) EDTA to other species. The evolution of visible brown coloration in solutions with $\geq 0.50 \mathrm{~mL}$ RedSol may also reflect development of $\mathrm{Co}$ (II)EDTA with its wavelength of maximum absorbance of $465 \mathrm{~nm}$. Other brown compounds have been shown to have absorbance maxima at wavelengths between 400 and $500 \mathrm{~nm}$ (Tang and Thompson, 2012; Wilson et al., 2016). Finally, increasing RedSol from 0.75 to $1.0 \mathrm{~mL}$ resulted in an apparent decline in absorbance across all wavelengths, suggesting dissociation of Co(III)EDTA into species that do not absorb visible light.

The Cr(III)EDTA showed maximum absorbance at $560 \mathrm{~nm}$ at the nearly neutral $\mathrm{pH}$ of the experimental media compared with $541 \mathrm{~nm}$ under slightly acidic conditions (Cerar, 2015). Accordingly, evaluations of $\mathrm{Cr}(\mathrm{III})$ EDTA were performed with absorbances determined at $560 \mathrm{~nm}$. In contrast to $\mathrm{Co}(\mathrm{III}) \mathrm{EDTA}$, the effect of RedSol additions $(P=0.32$; SED $=0.003)$ was only detected as a tendency for a linear decline (linear, $P=0.14$; other patterns of response: quadratic, $P=$ 0.29 ; cubic, $P=0.66)$ with a maximum of $8 \%$ decrease in absorbance (Figure 1b). The absorbances across wavelengths of visible light retained the basic pattern characteristic of $\mathrm{Cr}$ (III)EDTA but with absorbance declining with increasing additions of RedSol, and apparently more so at shorter wavelengths (Figure $2 \mathrm{~b}$ ). The redox potential at which $\mathrm{Cr}$ (III) is converted to $\mathrm{Cr}$ (II) is $-420 \mathrm{mV}$ (at $298.15 \mathrm{~K}$ and acidic pH; Bratsch, 1989), which is within the range of ORP values reported for the rumen. However, $\mathrm{Cr}$ (II) is easily oxidized back to $\mathrm{Cr}$ (III) through reactions with $\mathrm{O}_{2}$ and water depending on the acidity and anions present (Mandich, 1997). It is possible that some reduction of $\mathrm{Cr}$ (III) resulted in a
$\mathrm{Cr}$ (II)EDTA salt that is less stable than Cr(III)EDTA (respective stability constants of 13.6, Vinokurov and Bondar, 2003; and 23.6, Anderegg, 1977). As with Co(II)EDTA, if Cr(II)EDTA dissociated and did not reassociate as $\mathrm{Cr}$ (III)EDTA, the released ions would no longer be incorporated in colored compounds and absorbance would decline. A pattern of absorption across wavelengths for $\mathrm{Cr}$ (II)EDTA was not found in the literature.

The potential complete dissociation of $\mathrm{Co}(\mathrm{III}) \mathrm{EDTA}$ and partial dissociation of $\mathrm{Cr}$ (III)EDTA under reducing conditions lend themselves to explanation of the in vivo work of Shingfield et al. (2008). In that study, CoEDTA but not CrEDTA altered the fatty acid profile of milk
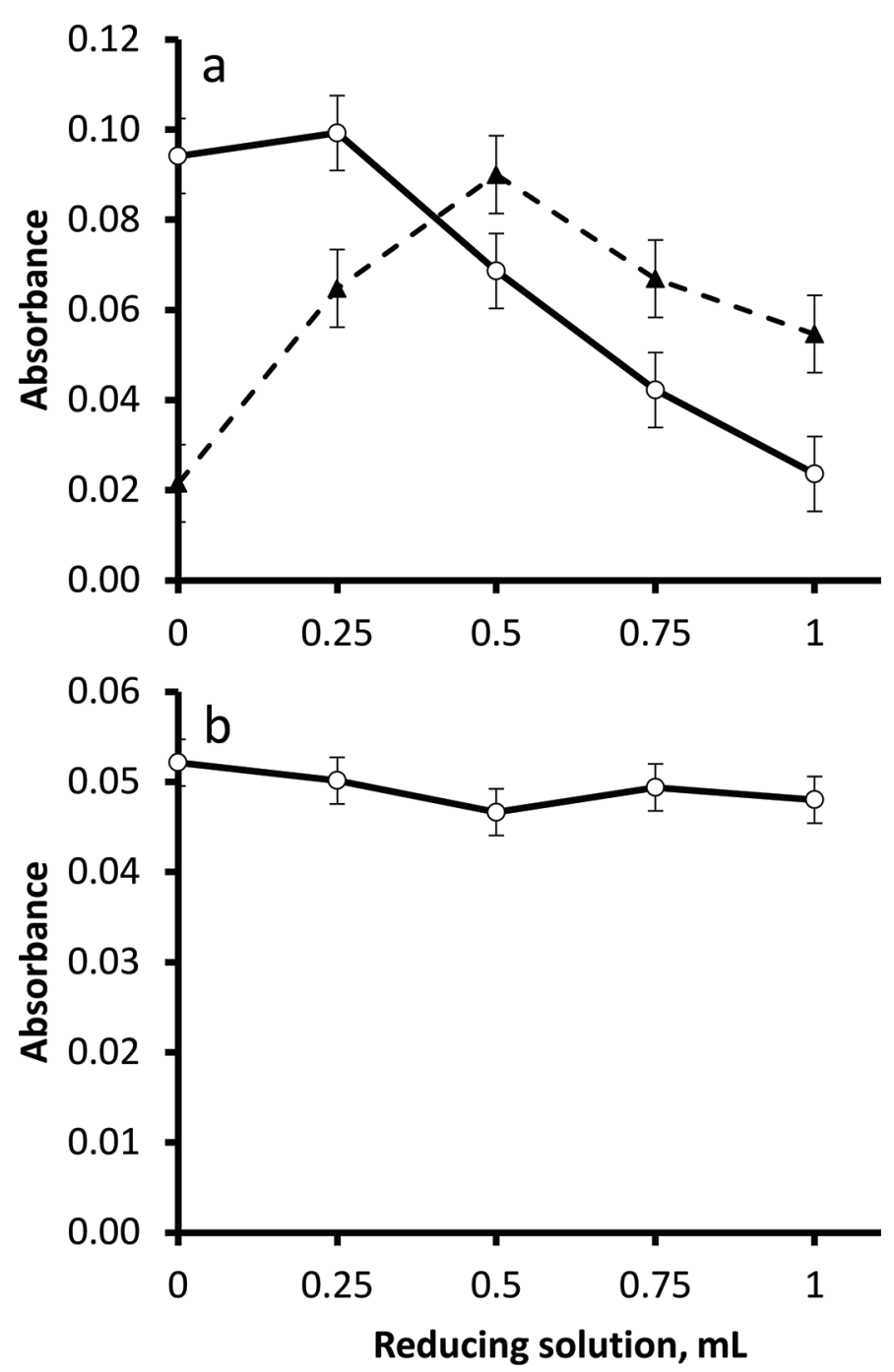

Figure 1. Absorbance values at wavelengths $(\lambda)$ of peak absorption for sample solutions of $\mathrm{Co}$ (III)EDTA and $\mathrm{Cr}$ (III)EDTA with different volumes of reducing solution added. Values are least squares means, and error bars represent SEM. (a) Co(III)EDTA absorbance at $\lambda=$ $535 \mathrm{~nm}, \mathrm{O}, \mathrm{SED}=0.008$ and $\lambda=465 \mathrm{~nm}, \mathbf{\Lambda} ; \mathrm{SED}=0.009$. (b) $\mathrm{Cr}(\mathrm{III})$ EDTA absorbance at $\lambda=560 \mathrm{~nm}, \mathrm{O}, \mathrm{SED}=0.003$. 


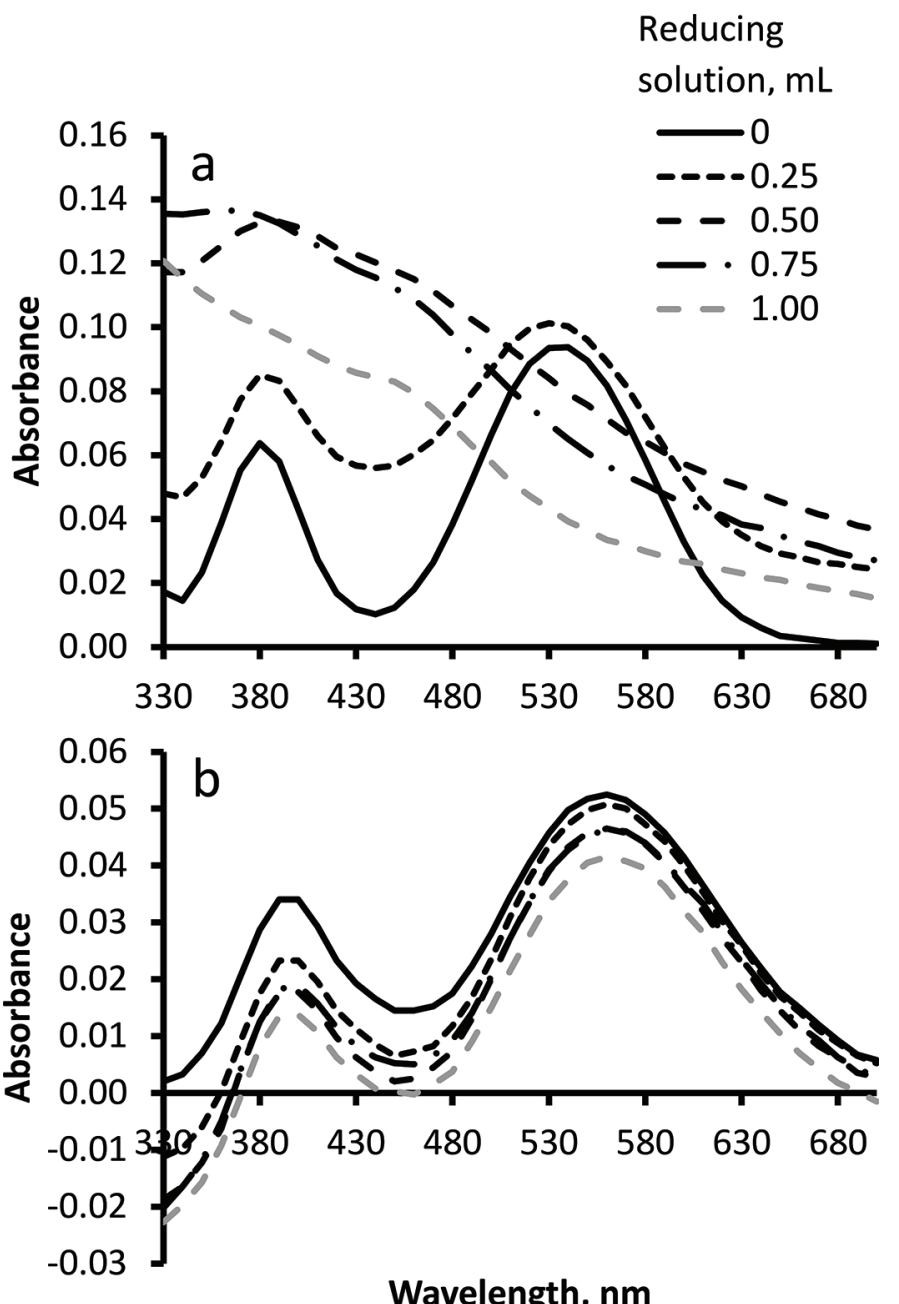

Figure 2. Wavescan absorbance data from wavelengths of 330 to $700 \mathrm{~nm}$ for (a) $\mathrm{Co}(\mathrm{III}) \mathrm{EDTA}$, and (b) $\mathrm{Cr}$ (III)EDTA sample solutions with different volumes of reducing solution added. Data are arithmetic averages of sample replicates from the second analytical run. Lines show wavelength scans for individual additions of reducing solution.

in lactating cows. In our previous work with unreduced, autoclaved, clarified rumen fluid, we had shown that CrEDTA and, to a lesser extent, CoEDTA could dissociate (14 and $4 \%$ by $24 \mathrm{~h}$, respectively) and potentially bind to phenolics or other ligands present (Hall and Van Soest, 2019b). If bound to other compounds, the minerals have the potential to remain nonreactive. However, unbound Co from extensively dissociated CoEDTA could generate the observed in vivo effects in dairy cattle through increased production of vitamin $\mathrm{B}_{12}$ analogs or as absorbed Co. A study in Wistar rats showed that vitamin $\mathrm{B}_{12}$ deficiency relative to folic acid adequacy or excess alters activity of hepatic desaturases (Wadhwani et al., 2012). An effectual $B_{12}$ deficiency could theoretically be produced if the infused CoEDTA dissociated and greatly increased the ruminally available Co and increased ruminal formation of vitamin $\mathrm{B}_{12}$ analogs (Kawashima et al., 1997). The analogs can inhibit enzyme activity by binding in place of coenzymes derived from $\mathrm{B}_{12}$ but without their activity (Lengyel et al., 1960). Alternatively, Co as cobalt acetate administered orally (3.5 g Co/d) or intravenously (175 mg Co/d) has been shown to alter mammary desaturase milk fatty acid desaturation products in dairy cows (Taugbøl et al., 2010); the oral dose of Co was approximately 1,500× recommendations (NRC, 2001), and the venous dose was $5 \%$ of the oral dose to be comparable to urinary Co excretion noted with markers. The carryover effect on mammary fatty acid desaturation products appears to vary by Co dose level, and may be greater than $10 \mathrm{~d}$ (Karlengen et al., 2013).

It seems less likely that CrEDTA is substantially dissociated in the gut. Dobson et al. (1976) investigated ruminal absorption of $\left[{ }^{51} \mathrm{Cr}\right]$ EDTA from the ventral sac of nonlactating dairy cows. In that study, eluate from urine applied to an anion exchange column "had a faint purple color" with a peak absorbance at $542 \mathrm{~nm}$. They concluded that the CrEDTA "passed from the rumen into the blood without appreciable degradation." This is suggestive, but not definitive, of a greater stability of CrEDTA as an intact molecule than CoEDTA.

\section{Consequences of Marker Dissociation}

To be useful in research, markers used to assess digesta kinetics or diet digestibility must not interfere with responses of the animal or the microbiome. Markers that become reactive under conditions found in the animal violate this standard and raise the question of whether responses seen in studies where they were used were a function of the treatments or interactions of treatments and the marker. This may also affect how treatment inferences can be applied to the larger population of animals that do not receive markers. It is clear from the in vivo data with Co(III)EDTA (Shingfield et al., 2008) and the results of the present study that $\mathrm{Co}$ (III)EDTA is not suitable as a digesta marker in ruminants because of its bioactivity and dissociation. The status of $\mathrm{Cr}$ (III)EDTA is slightly less clear. That it does partially dissociate in rumen fluid (Hall and Van Soest, 2019b) and under reducing conditions makes it less than ideal, and we do not know what the combination of reducing conditions and solutes in rumen fluid would do to the extent of dissociation. However, Shingfield et al. (2008) did not note an effect of CrEDTA on the specific measures they made in lactating dairy cattle, although the study was not suited to evaluation of lactation performance, having used 5 cows in a $5 \times 5$ Latin square. Reports of effects of CrEDTA on animal or microbial metabolism or diet digestibility 
were not found in the literature. Questions that require further investigation are whether any dissociated $\mathrm{Cr}$ recombines with indigestible compounds for it to stay inert and a marker in the liquid fraction, and whether CrEDTA affects other ruminal or animal responses of interest. With either EDTA marker, dissociation will release EDTA, which is not likely to degrade under anaerobic conditions (Tiedje, 1977) and could bind other minerals.

An alternative liquid digesta marker is polyethylene glycol (PEG; molecular weight 4,000 to 6,000) which was the earliest viable marker of liquid digesta investigated for use in nutrition studies (Shaffer and Critchfield, 1947). Polyethylene glycol of molecular weights 4,000 and 6,000 are reported not to be absorbed from the gut in rats or humans, although absorption of PEG of molecular weights 1,000 and 1,540 can be slight (Shaffer and Critchfield, 1947). Polyethylene glycol has been reported to not (Teeter and Owens, 1983) or minimally (Downes and McDonald, 1964) be recovered in urine in ruminants and has not been found to be appreciably degraded in the gut of ruminants (Teeter and Owens, 1983) or swine (Ishikawa, 1965). However, criticisms of PEG include that it interacts with tannins in feeds and has required analysis of undried samples using a turbidimetric method that is affected by technique. Feeds containing tannins, such as cottonseed hulls and a variety of tropical forages, may complex with PEG (Clark et al., 1972), thus removing the marker from the liquid pool. Use of PEG in diets with tanniniferous feeds is not recommended. Work has been done to stabilize the turbidimetric assay of Hydén (1955) and make it less sensitive to technique (Malawer and Powell, 1967), and PEG (molecular weight 6,000 ) has been reported to be detected by near-infrared reflectance spectroscopy in oven-dried feces from lactating dairy cows (Ahvenjärvi et al., 2018). Although PEG is not as ideal in its precision of measurement as the Co- and Cr-EDTA salts, it provides an alternative that may be less prone to absorption and bioactivity in the animal.

\section{CONCLUSIONS}

We conclude that $\mathrm{Co}$ (III)EDTA is not suitable for use as a liquid digesta marker in ruminants because of its dissociation under reducing conditions achievable in the rumen and the potential bioactive effect on animal and microbial responses. Chromium (III)EDTA shows less propensity for dissociation; however, it has not been fully investigated under reducing and chemical conditions present in the rumen to fully warrant its use. We suggest reexamining the suitability of available liquid digesta markers for use in ruminant research.

\section{ACKNOWLEDGMENTS}

Special thanks go to J. W. Pitas of USDA-ARS U.S. Dairy Forage Research Center (Madison, WI) for analytical assistance. This material is based upon work supported with funding from USDA-Agricultural Research Service (Washington, DC). Mention of any trademark or proprietary product in this paper does not constitute a guarantee or warranty of the product by the USDA or the Agricultural Research Service and does not imply its approval to the exclusion of other products that also may be suitable. The authors have not stated any conflicts of interest.

\section{REFERENCES}

Ahvenjärvi, S., L. Nyholm, J. Nousiainen, E. A. Mäntysaari, and M. Lidauer. 2018. Polyethylene glycol as an indigestible marker to estimate fecal output in dairy cows. J. Dairy Sci. 101:4245-4258. https://doi.org/10.3168/jds.2017-13337.

Anderegg, G. 1977. Critical survey of stability constants of EDTA complexes. IUPAC Chemical Data Series No. 14. Pergamon Press, Oxford, UK

AOAC International. 2005. Official Methods of Analysis. 18th ed. AOAC International, Washington, DC.

Bratsch, S. G. 1989. Standard electrode potentials and temperature coefficients in water at 298.15 K. J. Phys. Chem. Ref. Data 18:121. https://doi.org/10.1063/1.555839.

Cerar, J. 2015. Reaction between chromium (III) and EDTA ions: An overlooked mechanism of case study reaction of chemical kinetics. Acta Chim. Slov. 62:538-545. https://doi.org/10.17344/acsi.2015 .1492 .

Clark, J. L., F. G. Hembry, G. B. Thompson, and R. L. Preston. 1972. Ration effect on polyethylene glycol as a rumen marker. J. Dairy Sci. 55:1160-1164. https://doi.org/10.3168/jds.S0022 -0302(72)85639-X.

Dobson, A., A. F. Sellers, and V. H. Gatewood. 1976. Dependence of Cr-EDTA absorption from the rumen on luminal osmotic pressure. Am. J. Physiol. 231:1595-1600. https://doi.org/10.1152/ajplegacy .1976.231.5.1595.

Downes, A. M., and I. W. McDonald. 1964. The chromium-51 complex of ethylenediamine tetraacetic acid as a soluble rumen marker. Br. J. Nutr. 18:153-162. https://doi.org/10.1079/BJN19640015.

Faichney, G. J. 1993 Digesta flow. Pages 53-85 in Quantitative Aspects of Ruminant Digestion and Metabolism. J. M. Forbes and J. France, ed. CAB International, Wallingford, UK.

Fukushima, R. S., P. J. Weimer, and D. A. Kunz. 2003. Use of photocatalytic reduction to hasten preparation of culture media for saccharolytic Clostridium species. Braz. J. Microbiol. 34:22-26. https: //doi.org/10.1590/S1517-83822003000100006.

Goering, H. K., and P. J. Van Soest. 1970. Forage Fiber Analysis (Apparatus, Reagents, Procedures and Some Applications). Agric Handbook No. 379. ARS-USDA, Washington, DC.

Hall, M. B., and P. J. Van Soest. 2019a. Liquid digesta markers: A method for synthesis of crystallized chromium-EDTA and comparison of its degree of complexation with an uncrystallized preparation. Anim. Feed Sci. Technol. 253:32-38. https://doi.org/10 .1016/j.anifeedsci.2019.05.002.

Hall, M. B., and P. J. Van Soest. 2019b. Stability of the liquid markers chromium (III) and cobalt (III)-EDTA in autoclaved, clarified rumen fluid. J. Dairy Sci. 102:7049-7058. https://doi.org/10.3168/ jds.2018-15768.

Hamm, R. E. 1953. Complex ions of chromium. IV. The ethylenediaminetetraacetic acid complex with chromium (III). J. Am. Chem. Soc. 75:5670-5672. https://doi.org/10.1021/ja01118a059. 
Hedrick, C. E. 1965. Formation of the chromium-EDTA complex: An undergraduate kinetics experiment. J. Chem. Educ. 42:479-480. https://doi.org/10.1021/ed042p479.

Huang, Y., J. P. Marden, C. Julien, and C. Bayourthe. 2018. Redox potential: An intrinsic parameter of the rumen environment. J. Anim. Physiol. Anim. Nutr. (Berl.) 102:393-402. https://doi.org/ 10.1111/jpn.12855.

Hydén, S. 1955. A turbidimetric method for the determination of higher polyethylene glycols in biological materials. Kungl. Lantbrukshögskolans Annaler 22:139-145.

Ishikawa, S. 1965. Degradation of polyethylene glycol in the digestive tract. Agric. Biol. Chem. 29:173-180. https://doi.org/10.1080/ 00021369.1965.10858374.

Karlengen, I. J., O. Taugbøl, B. Salbu, A. H. Aastveit, and O. M. Harstad. 2013. Effect of different levels of supplied cobalt on the fatty acid composition of bovine milk. Br. J. Nutr. 109:834-843. https://doi.org/10.1017/S0007114512002243.

Kawashima, T., P. R. Henry, D. G. Bates, C. B. Ammerman, R. C. Littell, and J. Price. 1997. Bioavailability of cobalt sources for ruminants. 3. In vitro ruminal production of vitamin $\mathrm{B}_{12}$ and total corrinoids in response to different cobalt sources and concentrations. Nutr. Res. 17:975-987. https://doi.org/10.1016/S0271 $-5317(97) 00062-6$.

Lengyel, P., R. Mazumder, and S. Ochoa. 1960. Mammalian methylmalonyl isomerase and vitamin B12 coenzymes. Proc. Natl. Acad. Sci. USA 46:1312-1318. https://doi.org/10.1073/pnas.46.10.1312.

Malawer, S. J., and D. W. Powell. 1967. An improved turbidimetric analysis of polyethylene glycol utilizing an emulsifier. Gastroenterology 53:250-256. https://doi.org/10.1016/S0016-5085(19)34232 $-5$

Mandich, N. V. 1997. Chemistry and theory of chromium deposition: Part I - Chemistry. Plat. Surf. Finish. 84:108-115.

NRC. 2001. Nutrient Requirements of Dairy Cattle. 7th rev. ed. National Academies Press, Washington, DC.

Ogino, H., and K. Ogino. 1983. Redox potentials and related parameters of cobalt (III/II) complexes containing aminopolycarboxylates. Inorg. Chem. 22:2208-2211. https://doi.org/10.1021/ ic00157a023.

Paraneeiswaran, A., S. K. Shukla, V. S. Sathyaseelan, and T. S. Rao. 2015. A spectrophotometric method for the determination Co-EDTA complexes. Int. J. Appl. Sci. Biotechnol. 3:584-587. https:// doi.org/10.3126/ijasbt.v3i4.13182.

Qin, C., D. Bu, P. Sun, X. Zhao, P. Zhang, and J. Wang. 2017. Effects of corn straw or mixed forage diet on rumen fermentation parameters of lactating cows using a wireless data logger. Anim. Sci. J. 88:259-266. https://doi.org/10.1111/asj.12616.

Richter, M., L. Krížová, and J. Trináctý. 2010. The effect of individuality of animal on diurnal pattern of $\mathrm{pH}$ and redox potential in the rumen of dry cows. Czech J. Anim. Sci. 55:401-407. https://doi .org/10.17221/1695-CJAS.

Shaffer, C. B., and F. H. Critchfield. 1947. The absorption and excretion of the solid polyethylene glycols ("Carbowax" compounds). J. Pharm. Sci. 36:152-157. https://doi.org/10.1002/jps.3030360507.
Shingfield, K. J., A. Arölä, S. Ahvenjärvi, A. Vanhatalo, V. Toivonen, J. M. Griinari, and P. Huhtanen. 2008. Ruminal infusions of cobalt-EDTA reduce mammary $\Delta 9$-desaturase index and alter milk fatty acid composition in lactating cows. J. Nutr. 138:710-717. https://doi.org/10.1093/jn/138.4.710.

Tang, H., and J. E. Thompson. 2012. Light absorbing products form during the aqueous phase reaction of phenolic compounds in the presence of nitrate and nitrite with UV illumination. Open J. Air Pollut. 01:13-21. https://doi.org/10.4236/ojap.2012.12002.

Taugbøl, O., I. J. Karlengen, B. Salbu, A. H. Aastveit, and O. M. Harstad. 2010. Intravenous injections of cobalt reduce fatty acid desaturation products in milk and blood of lactating cows. J. Anim. Physiol. Anim. Nutr. (Berl.) 94:635-640. https://doi.org/ 10.1111/j.1439-0396.2009.00950.x.

Teeter, R. G., and F. N. Owens. 1983. Characteristics of water soluble makers for measuring rumen liquid volume and dilution rate. J. Anim. Sci. 56:717-728. https://doi.org/10.2527/jas1983.563717x.

Tiedje, J. M. 1977. Influence of environmental parameters on EDTA biodegradation in soils and sediments. J. Environ. Qual. 6:21-26. https://doi.org/10.2134/jeq1977.00472425000600010006x.

Udén, P., P. E. Colucci, and P. J. Van Soest. 1980. Investigation of chromium, cerium and cobalt as markers in digesta. Rate of passage studies. J. Sci. Food Agric. 31:625-632. https://doi.org/10 $.1002 /$ jsfa. 2740310702 .

Vinokurov, E. G., and V. V. Bondar. 2003. Prediction of stability constants for $\mathrm{Cr}$ (III) and $\mathrm{Cr}(\mathrm{II})$ complexes. Russ. J. Coord. Chem. 29:66-72. https://doi.org/10.1023/A:1021851219309.

Wadhwani, N. S., R. R. Manglekar, K. D. Dangat, A. V. Kulkarni, and S. R. Joshi. 2012. Effect of maternal micronutrients (folic acid, vitamin $\mathrm{B}_{12}$ ) and omega 3 fatty acids on liver fatty acid desaturases and transport proteins in Wistar rats. Prostaglandins Leukot. Essent. Fatty Acids 86:21-27. https://doi.org/10.1016/j.plefa.2011 .10 .010 .

Waghorn, G. C. 1991. Electronegativity and redox potential of rumen digesta in situ in cows eating fresh lucerne. N. Z. J. Agric. Res. 34:359-361. https://doi.org/10.1080/00288233.1991.10417676.

Weisbjerg, M. R., T. Hvelplund, and B. M. Bibby. 1998. Hydrolysis and fermentation rate of glucose, sucrose and lactose in the rumen. Acta. Agric. Scand. A Anim. Sci. 48:12-18.

Wilson, A., S. Prabukumar, G. Sathishkumar, and S. Sivaramakrishnan. 2016. Aspergillus flavus mediated silver nanoparticles synthesis and evaluaton of its antimicrobial activity against different human pathogens. Int. J. App. Pharm. 8:43-46. https://doi.org/ 10.22159/ijap.2016v8i4.13930.

Xue, Y., and S. J. Traina. 1996. Oxidation kinetics of Co(II)-EDTA in aqueous and semi-aqueous goethite suspensions. Environ. Sci. Technol. 30:1975-1981. https://doi.org/10.1021/es950715f.

\section{ORCIDS}

Mary Beth Hall @ https://orcid.org/0000-0002-5460-3208 